\title{
Nota Técnica da Sociedade Brasileira de Nutrição Oncológica sobre os Cuidados Nutricionais em Oncologia frente à Pandemia de Covid-19
}

doi: https://doi.org/10.32635/2176-9745.RBC.2020v66nTemaAtual.1011

\author{
Technical Note from the Brazilian Society of Oncology Nutrition on Nutritional Oncology Care because of COVID-19 Pandemic \\ Nota Técnica de la Sociedad Brasileña de Nutrición Oncológica sobre Cuidados Nutricionales en Oncología ante la Pandemia \\ de Covid-19
}

Renata Brum Martucci'; Ana Maria Calabria Cardoso ${ }^{2}$; Carin Weirich Gallon ${ }^{3}$; Erika Simone Coelho Carvalho4; Izabella Fontenelle de Menezes Freitas ${ }^{5}$; Lilianne Carvalho Santos Roriz ${ }^{6}$; Luciana Zuolo Coppini ${ }^{7}$; Luciane Beitler da Cruz ${ }^{8}$; Maria Amélia Dantas ${ }^{9}$; Maria Lúcia Varjão da Costa ${ }^{10}$; Nádia Dias Gruezo ${ }^{11}$; Viviane Dias Rodrigues ${ }^{12}$; Nivaldo Barroso de Pinho ${ }^{13}$

\section{INTRODUÇÃO}

Coronavírus é uma família de vírus que causam infecçôes respiratórias. O novo coronavírus (2019-nCoV) foi descoberto no final de 2019 na China, sendo a doença considerada pela Organização Mundial da Saúde (OMS) uma pandemia, chamada de Covid-19 (coronavirus disease 2019). Sars-CoV-2 é a sigla para o vírus da síndrome respiratória aguda grave do coronavírus 2 (severe acute respiratory syndrome coronavirus 2), principal característica da infecção, que se espalha principalmente pelo trato respiratório, por gotículas, secreçōes respiratórias e contato direto ${ }^{1}$.

O paciente com câncer, seja em tratamento ou o sobrevivente, possui características que podem propiciar as formas mais graves da doença e maior mortalidade, entre estas, podem ser citadas maior idade, presença de comorbidades, hipoalbuminemia, tratamentos imunossupressores e tabagismo ${ }^{2-6}$. Alguns sintomas como náuseas, vômitos, diarreia, anosmia, disgeusia já foram relatados em pacientes com Covid-197,8. Sabe-se que a presença desses sintomas está fortemente associada com a desnutrição na população oncológica brasileira9 .

Um documento recente sobre o fluxo de assistência nutricional de pacientes com Covid-19, em unidade hospitalar, considera que idosos $\geq 65$ anos, adultos com índice da massa corporal (IMC) $<20,0 \mathrm{~kg} / \mathrm{m}^{2}$, pacientes com risco alto ou lesão por pressão, imunossuprimidos, inapetentes, com diarreia persistente, histórico de perda de peso, doença pulmonar obstrutiva crônica (DPOC), asma, pneumopatias estruturais, cardiopatias, incluindo hipertensão arterial importante, diabetes insulinodependente e insuficiência renal sáo considerados em risco nutricional ${ }^{8}$.

Segundo o Instituto Nacional de Câncer José Alencar Gomes da Silva (INCA), pessoas com câncer em tratamentos em quimioterapia, radioterapia, que tenham feito cirurgia há menos de um mês ou que façam uso de medicamentos imunossupressores, fazem parte do grupo de risco $^{10}$.

${ }^{1}$ Doutora. Nutricionista. Coordenadora-Científica e Membro Fundador da Sociedade Brasileira de Nutrição Oncológica (SBNO). Seção de Nutrição e Dietética do Hospital de Câncer I ( $\mathrm{HCl}$ ) do Instituto Nacional de Câncer José Alencar Gomes da Silva (INCA). Professor-Associado do Instituto de Nutrição da Universidade do Estado do Rio de Janeiro (Uerj). Rio de Janeiro (RJ), Brasil. Orcid iD: https://orcid.org/0000-0002-3354-4229

${ }^{2}$ Mestre. Nutricionista. Membro Fundador da SBNO. Comitê de Ética em Pesquisa. Núcleo de Pesquisa em Oncologia. Universidade Federal do Pará. Belém (PA), Brasil. Orcid iD: https://orcid.org/0000-0002-0673-7711

${ }^{3}$ Doutora. Nutricionista. Membro Fundador da SBNO. Universidade de Caxias do Sul. Rio Grande do Sul (RS), Brasil. Orcid iD: https://orcid.org/0000-0001-7874-1595 ${ }^{4}$ Mestre. Nutricionista. Coordenadora de Ensino e Membro Fundador da SBNO. Onco-Hematologia do Hospital Governador Israel Pinheiro. Instituto de Previdência dos Servidores do Estado de Minas Gerais. Minas Gerais (MG), Brasil. Orcid iD: https://orcid.org/0000-0001-7465-7417

${ }^{5}$ Especialista. Nutricionista. Membro Fundador da SBNO. Gerente de Nutrição da Associação Piauiense de Combate ao Câncer Alcenor Almeida, Hospital São Marcos. Teresina (PI), Brasil. Orcid iD: https://orcid.org/0000-0001-6826-9581

${ }^{6}$ Especialista. Nutricionista. Membro Fundador da SBNO. Goiânia (GO), Brasil. Orcid iD: https://orcid.org/0000-0003-0910-6373

${ }^{7}$ Mestre. Nutricionista. Membro Fundador da SBNO. Centro Integrado de Nutrição. São Paulo (SP), Brasil. Orcid iD: https://orcid.org/0000-0002-5499-9521

${ }^{8}$ Doutora. Nutricionista. Membro Fundador da SBNO. Hospital das Clínicas de Porto Alegre. Porto Alegre (RS), Brasil. Orcid iD: https://orcid.org/0000-0002-2977-0696

${ }^{9}$ Especialista. Nutricionista. Membro Fundador da SBNO. Liga Norte Riograndense Contra o Câncer. Natal (RN), Brasil. Orcid iD: https://orcid.org/0000-0002-5630-4287

${ }^{10}$ Mestre. Nutricionista. Membro Fundador da SBNO. Hospital Aristides Maltez. Salvador (BA), Brasil. Orcid iD: https://orcid.org/0000-0002-5648-6738

${ }^{11}$ Doutora. Nutricionista. Membro Fundador da SBNO. Gerência da Assistência Complementar e Essencial do Hospital da Criança de Brasília José Alencar. Brasília (DF), Brasil. Orcid iD: https://orcid.org/0000-0001-6355-7314

${ }_{12}$ Mestre. Nutricionista. Vice-Presidente da SBNO. Chefe da Seção de Nutrição e Dietética do HCI/INCA. Rio de Janeiro (RJ), Brasil. Orcid iD: https://orcid.org/00000003-2243-438X

${ }^{13}$ Doutor. Nutricionista. Presidente da SBNO. Coordenador da Divisão de Apoio Técnico do HCI/INCA. Rio de Janeiro (RJ), Brasil. Orcid iD: https://orcid.org/00000002-1438-168X

Endereço para correspondência: Renata Brum Martucci. Seção de Nutrição e Dietética do INCA. Praça Cruz Vermelha, 23 - 5ªndar - Centro. Rio de Janeiro (RJ), Brasil. CEP 20230-130. E-mail: renata.martucci@inca.gov.br 


\section{RECOMENDAÇÕES}

Embora as evidências reais estejam focadas no gerenciamento geral da Covid-19, pouco se sabe sobre o suporte nutricional durante a internação hospitalar. A falta de procedimentos nutricionais pode, por sua vez, prolongar a recuperação dos pacientes e aumentar ainda mais as complicaçóes infecciosas ${ }^{11}$.

O Conselho Federal de Nutricionistas (CFN) em seu documento recente recomenda: "Os nutricionistas devem analisar cautelosamente o cenário e os normativos locais para entender a gravidade do problema e considerar as importantes limitaçóes impostas pela ausência da avaliação e contato físicos com o cliente/paciente/usuário para avaliar a melhor modalidade de atendimento para a adequada assistência nutricional" ${ }^{12}$. Nesse sentido, o nutricionista deve avaliar a necessidade de manutenção dos atendimentos presenciais, seja ambulatório, consultório ou em domicílio.

Assim como o $\mathrm{CFN}^{12}$ se posicionou, a Sociedade Brasileira de Nutrição Oncológica (SBNO) ratifica que não há evidências científicas que sustentem a existência de alimentos e ou fórmulas com ação protetora contra o Covid-19. A alimentação saudável e equilibrada contribui para um sistema imunológico mais eficiente.

O tratamento oncológico está mantido e, consequentemente, todos os protocolos de assistência nutricional ${ }^{13,14}$. O objetivo da presente Norma Técnica é garantir as melhores condiçôes de saúde dos pacientes e minimizar os riscos de infecção dos profissionais, pacientes e familiares.

\section{AtENDIMENTO AMBULATORIAL}

- Os atendimentos ambulatoriais de pacientes, pré ou pós-tratamento oncológico e em grupo, devem ser suspensos durante a pandemia, ou realizados on-line, podendo os pacientes ser contactados por telefone. De acordo com o CFN, a assistência nutricional, exclusivamente por meio não presencial, pode ser realizada, incluindo consultas de avaliação e diagnóstico nutricional.

- Pacientes em tratamento oncológico ou que comparecerem à unidade por demanda espontânea devem ter o acompanhamento nutricional mantido, respeitando todas as normas de biossegurança preconizadas para proteção pessoal do profissional e do paciente.

- Pacientes com sintomas da Covid-19 devem ser orientados a seguir os cuidados preconizados pelas autoridades sanitárias e não devem ser atendidos.

- Reorganizar a sala de espera, deixando espaço de 1 metro entre as cadeiras e disponibilizar materiais educativos (cartazes) sobre cuidados de higiene e equipamentos de proteção individual (EPI) e álcool em gel.

- Manter intervalo suficiente entre os atendimentos para a correta higienização do local, conforme orientaçáo institucional.

- O local de atendimento deve ser adequado, ventilado ou com portas abertas, e retirado todo material ou equipamento náo essencial, mesas e cadeiras com distância mínima de 1,5 metro entre o profissional e o paciente.

- O uso de máscaras pelo profissional e pelo paciente é obrigatório.

- Se possível, disponibilize protetor de calçados descartável para a entrada na sala.

- As orientações nutricionais podem ser enviadas por e-mail ou na forma de mensagem de texto.

- A necessidade de uso de materiais para avaliação nutricional (balança, fita, adipômetro e outros) deve ser avaliada com cautela e, caso seja necessário, devem ser higienizados corretamente.

\section{UNIDADE HOSPITALAR}

- O profissional deve usar medidas de higiene pessoal e EPI adequados durante a permanência na unidade hospitalar, seguindo as orientaçóes da sua Comissão de Controle de Infecção Hospitalar (CCIH).

- Manter a triagem nutricional dos pacientes adultos, idosos e pediátricos, conforme o protocolo da unidade sempre que possível. Dar preferência as ferramentas que podem ser preenchidas pelo próprio paciente ou na forma de entrevistas realizadas por telefone.

- Caso náo seja possível realizar a triagem nutricional, considere todos os pacientes oncológicos com Covid-19 confirmados como em risco nutricional. Devem-se manter as recomendaçóes do Consenso Nacional de Nutriçáo Oncológica para a instituiçáo da terapia nutricional precoce em pacientes oncológicos em risco nutricional ${ }^{15,16}$.

- Não realizar avaliação nutricional dos pacientes suspeitos ou confirmados, com o objetivo de evitar contato físico.

- Na reavaliação diária da ingestão alimentar e sintomatologia, usar dados secundários de prontuário, de contato telefônico com o paciente e do intermédio de membros da equipe multiprofissional que já estejam em contato direto com esses pacientes.

- Evitar entrar em unidades de terapia intensiva, com pacientes confirmados. Devem-se manter as recomendaçóes do Consenso Nacional de Nutrição Oncológica para a instituição da terapia nutricional em pacientes oncológicos críticos ${ }^{15,16}$. 
- Na linha de produção dos alimentos aos pacientes internados, considera-se fundamental o atendimento fiel às Boas Práticas de Fabricação e de Manipulação de Alimentos neste momento, de forma a continuar a garantir a entrega de alimentos seguros ${ }^{17}$.

\section{PESQUISA CLÍNICA}

- Suspender as coletas de dados de pesquisas em andamento ou aprovadas pelo Comitê de Ética em Pesquisa de pacientes que não estão em tratamento oncológico ou que aumentem o fluxo de pacientes na unidade.

- Apoiar o desenvolvimento de ferramentas tecnológicas para o contato com os pacientes em isolamento.

- Incentivar o desenvolvimento de projetos de pesquisa sobre aspectos envolvendo nutriçáo, câncer e Covid-19, com coleta de dados retrospectiva após controle da pandemia.

\section{CONCLUSÃO}

$\mathrm{O}$ atendimento presencial e a avaliação nutricional devem ser reavaliados em razão do cenário de pandemia. Os cuidados nutricionais sáo essenciais durante o tratamento oncológico. $\mathrm{O}$ nutricionista deve preservar a integridade do paciente oncológico tanto no contexto de prevenção da Covid-19 quanto na prevenção aos fatores de risco para desnutriçáo, de acordo com o Consenso Nacional de Nutrição Oncológica.

\section{CONTRIBUIÇÕES}

Todos os autores contribuíram igualmente na concepçáo, no levantamento bibliográfico, na redação, na revisão crítica e aprovaram a versão final a ser publicada.

\section{DECLARAÇÃO DE CONFLITO DE INTERESSES}

Nada a declarar.

\section{FONTES DE FINANCIAMENTO}

Não há.

\section{REFERÊNCIAS}

1. Guo YR, Cao QD, Hong ZS, et al. The origin, transmission and clinical therapies on coronavirus disease 2019 (COVID-19) outbreak - an update on the status. Mil Med Res. 2020;7(1):11. doi: https://doi. org/10.1186/s40779-020-00240-0

2. Liang W, Guan W, Chen R, et al. Cancer patients in SARS-CoV-2 infection: a nationwide analysis in China.
Lancet Oncol. 2020;21(3):335-37. doi: https://doi. org/10.1016/S1470-2045(20)30096-6

3. Wang $\mathrm{H}$, Zhang L. Risk of COVID-19 for patients with cancer. Lancet Oncol. 2020;21(4):E181. doi: https://doi. org/10.1016/S1470-2045(20)30149-2

4. Zhang L, Zhu F, Xie L, et al. Clinical characteristics of COVID-19-infected cancer patients: a retrospective case study in three hospitals within Wuhan, China. Ann Oncol. 2020:pii:S0923-7534(20)36383-3. doi: https:// doi.org/10.1016/j.annonc.2020.03.296 Epub ahead of print.

5. Desai A, Sachdeva S, Parekh T, et al. COVID-19 and cancer: lessons from a pooled meta-analysis. JCO Glob Oncol. 2020;6:557-559. doi: https://doi.org/10.1200/ GO.20.00097

6. Zhou F, Yu T, Du R, et al. Clinical course and risk factors for mortality of adult inpatients with COVID-19 in Wuhan, China: a retrospective cohort study. Lancet. 2020;395(10229):1054-62. doi: https://doi. org/10.1016/S0140-6736(20)30566-3

7. Guan WJ, Ni ZY, Hu Y, et al. Clinical characteristics of coronavirus disease 2019 in China. N Engl J Med. 2020;382:1708-20. doi: https://doi.org/10.1056/ NEJMoa2002032

8. Piovacari SMF, Santos GFCG, Santana GA, et al. Fluxo de assistência nutricional para pacientes admitidos com COVID-19 e SCOVID-19 em unidade hospitalar. BRASPEN J [Internet]. 2020 [acesso $2020 \mathrm{abr}$ 26];35(1):6-8. Disponível em: https://66b28c71-9a364ddb-9739-12f146d519be.usrfiles.com/ugd/66b28c_2 f5d298499184d22b2655dff908f58c9.pdf

9. de Pinho NB, Martucci RB, Rodrigues VD, et al. Malnutrition associated with nutrition impact symptoms and localization of the disease: results of a multicentric research on oncological nutrition. Clin Nutr. 2019 Jun 1;38(3):1274-9. doi: https://doi.org/10.1016/j. clnu.2018.05.010

10. Instituto Nacional de Câncer José Alencar Gomes da Silva [Internet]. Rio de Janeiro: INCA; c2020. Perguntas frequentes: câncer e coronavírus (Covid-19); [2020]. [acesso 2020 abr 26]. Disponível em: https://www. inca.gov.br/perguntas-frequentes/cancer-e-coronaviruscovid-19

11. Cintoni M, Rinninella E, Annetta MG, et al. Nutritional management in hospital setting during SARS-CoV-2 pandemic: a real-life experience. Eur J Clin Nutr. 2020 Apr 06. doi: https://doi.org/10.1038/s41430-020-0625-4

12. Conselho Federal de Nutricionistas. Recomendaçôes do CFN: boas práticas para a atuação do nutricionista e do técnico em nutrição e dietética durante a pandemia do novo coronavírus (COVID-19) [Internet]. 3 ed. rev. ampl. Brasília, DF: CFN; 2020. [acesso 2020 abr 26]. Disponível em: https://www.cfn.org.br/wp-content/ uploads/2020/03/nota_coronavirus_3-1.pdf 
13. Secretaria Estadual de Saúde do Rio de Janeiro. Resoluçấo SES no 2004, de 18 de março de 2020 [Internet]. Regulamenta as atividades ambulatoriais nas unidades de saúde públicas, privadas e universitários com atendimento ambulatorial e no Estado do Rio de Janeiro. Diário Oficial do Estado do Rio de Janeiro, Rio de Janeiro; 2020 mar 19. Ano 46, n. 51, Part I, p. 14. [acesso 2020 abr 26]. Disponível em: https://www. legisweb.com.br/legislacao/?id=390927

14. Ministério da Saúde (BR). Diretrizes para diagnóstico e tratamento da COVID-19 [Internet]. Brasília, DF: Ministério da Saúde; 2020 abr 06. [acesso 2020 abr 04]. Disponível em: https://portalarquivos.saude.gov. br/images/pdf/2020/April/07/ddt-covid-19.pdf

15. Instituto Nacional de Câncer José Alencar Gomes da Silva, Coordenação Geral de Gestão Assistencial, Hospital do Câncer I, Serviço de Nutrição e Dietética. Consenso nacional de nutriçáo oncológica. 2 ed. rev. ampl. atual. Rio de Janeiro: INCA; 2015.

16. Instituto Nacional de Câncer José Alencar Gomes da Silva, Coordenação Geral de Gestão Assistencial, Hospital do Câncer I, Serviço de Nutrição e Dietética. Consenso nacional de nutrição oncológica. 2 ed. rev. ampl. atual. Vol. 2, Rio de Janeiro: INCA; 2016

17. Agência Nacional de Vigilância Sanitária. Nota Técnica No 18: Covid-19 e as boas práticas de fabricaçáo e manipulação de alimentos [Internet]. Brasília, DF: ANVISA; 2020 abr 06. [acesso 2020 abr 04]. Disponível em: http://portal.anvisa.gov.br/ documents/219201/4340788/NT+18.2020+-+Boas+Pr $\%$ C3\%A1ticas+e+Covid+19/78300ec1-ab80-47fc-ae0a$4 d 929306 \mathrm{e} 38 \mathrm{~b}$ 